\title{
Design, Fabrication, and Testing of a Capsule With Hybrid Locomotion for Gastrointestinal Tract Exploration
}

\author{
Massimiliano Simi, Pietro Valdastri, Member, IEEE, Claudio Quaglia, Member, IEEE, \\ Arianna Menciassi, Member, IEEE, and Paolo Dario, Fellow, IEEE
}

\begin{abstract}
This paper describes a novel solution for the active locomotion of a miniaturized endoscopic capsule in the gastrointestinal (GI) tract. The authors present the design, development, and testing of a wireless endocapsule with hybrid locomotion, where hybrid locomotion is defined as the combination between internal actuation mechanisms and external magnetic dragging. The capsule incorporates an internal actuating legged mechanism, which modifies the capsule profile, and small permanent magnets, which interact with an external magnetic field, thus imparting a dragging motion to the device. The legged mechanism is actuated whenever the capsule gets lodged in collapsed areas of the GI tract. This allows modification of the capsule profile and enables magnetic dragging to become feasible and effective once again. A key component of the endoscopic pill is the internal mechanism, endowed with a miniaturized brushless motor and featuring compact design, and adequate mechanical performance. The internal mechanism is able to generate a substantial force, which allows the legs to open against the intestinal tissue that has collapsed around the capsule body. An accurate simulation of the performance of the miniaturized motor under magnetic fields was carried out in order to define the best configuration of the internal permanent magnets (which are located very close to the motor) and the best tradeoff operating distance for the external magnet, which is responsible for magnetically dragging the capsule. Finally, a hybrid capsule was developed generating $3.8 \mathrm{~N}$ at the tip of the legged mechanism and a magnetic link force up to $135 \mathrm{mN}$. The hybrid capsule and its wireless control were extensively tested in vitro, ex vivo, and in vivo, thus confirming fulfilment of the design specifications and demonstrating a good ability to manage collapsed areas of the intestinal tract.
\end{abstract}

Index Terms - Capsule endoscopy, endoscopic capsule, magnetic locomotion, robotic surgery.

\section{INTRODUCTION}

C URRENT technologies in biomedical engineering focus on reducing pain during diagnostic procedures, thus enabling extensive screening among individuals who are more likely to develop cancer. This has yet to be achieved for the

Manuscript received June 25, 2009; revised November 30, 2009. First published February 25, 2010; current version published March 31, 2010 Recommended by Guest Editor J. P. Desai. This work was supported in part by the Intelligent Microsystem Center, Korean Institute of Science and Technology, South Korea, and in part by the European Commission in the framework of VECTOR FP6 under European Project EU/IST-2006-033970.

The authors are with the Center for Research in Microengineering Laboratory, Scuola Superiore Sant'Anna, 56127 Pisa, Italy (e-mail: m.simi@ sssup.it pietro@sssup.it; c.quaglia@crim.sssup.it; arianna@sssup.it; dario@sssup.it).

Color versions of one or more of the figures in this paper are available online at http://ieeexplore.ieee.org.

Digital Object Identifier 10.1109/TMECH.2010.2041244 inspection of the lower gastrointestinal (LGI) tract, where long and semiflexible video probes are introduced into the oral or rectal cavities. This approach provides a detailed and reliable diagnosis and currently represents the best available technique for managing pathological diseases of the digestive tract. The major drawback is the procedure's invasiveness, making it frequently ill-tolerated by patients.

In order to reduce invasiveness, the use of wireless capsule endoscopy for screening procedures [1]-[3] has increased over recent years. This approach has proved to be mostly effective for the small bowel, with different endoscopic capsules already available in the market from several suppliers worldwide, such as Given Imaging [4], Olympus [5], and Intromedic [6]. These devices are mainly composed of a $640 \times 480$ pixel resolution camera, a LED-based illumination system, a data transmitter, and a suitable battery, which guarantees sufficient operative lifetime. All these components are incorporated in a pill with size ranging from the Intromedic MiRo (a diameter $(\phi)$ of $10.8 \mathrm{~mm}$ and length $(L)$ of $24 \mathrm{~mm}$ ), to the Given Imaging PillCam Colon $(\phi=11 \mathrm{~mm}, L=31 \mathrm{~mm})$. Despite capsule endoscopes are well-tolerated by patients, the diagnostic outcomes are not fully reliable. This is mainly due to the lack of active and teleoperated locomotion, because the pills move by simply exploiting GI peristalsis. Consequently they cannot stop, turn, or change direction to review and verify suspicious lesions, as in the case of traditional endoscopes. Furthermore, without proper tissue distension, obtained through air insufflation (which is standard practice today), interesting spots behind intestinal folds could be missed.

The ideal capsule for GI tract access should combine small size (small enough to swallow and to move by natural peristalsis) and active locomotion (ability to stop, and possibly, to move against peristalsis), and not require inflation of the colon, which is the source of much of the pain experienced during the traditional procedure [7]. If LGI endoscopy were to become a screening procedure due to such a system, this would significantly enhance public health and improve cancer screening. With regard to this, it is important to point out that yearly death rates from cancer dropped by 27\% from 1975 to 2005, largely due to prevention and early diagnosis [8].

The development of a wireless capsule that is capable of moving actively inside the GI tract while teleoperated by the endoscopist is a challenging task, given the features peculiar to the intestinal environment: unstructured, highly deformable, 
slippery (i.e., covered by a layer of lubricating mucus), and partially collapsed [9], [10]. Recent works addressing this issue, propose solutions where the source of active locomotion can be either external or internal to the patient's body.

External active locomotion can be obtained by using magnets inside the capsule coupled with an external magnetic field, generated either by coils [5], [11] or permanent magnets [12]-[15]. Controlled variations of the external magnetic field drag and orient the capsule in the desired direction. This approach does not require supply from the on-board battery, thus saving available energy for other different functions. The most critical limitation of this approach is that magnetic locomotion is successful only if the tissue is distended, instead, in the collapsed regions of the LGI tract, friction prevents effective control. Moreover, this approach does not allow per se tissue distension, thus preventing a complete view of the lumen walls.

The alternative approach consists in placing one or more actuators inside the video pill in order to implement an internal locomotion system. By correctly designing the locomotion mechanism, an effective relative motion between the capsule and the surrounding tissue can be achieved. Several devices with embedded actuators and mechanisms have been proposed, providing satisfactory locomotion performance inside hollow cavities [16]-[20]. In particular, studies on legged capsules for LGI endoscopy have demonstrated that two independent leg sets promote effective motion in the gut [21], and that the maximization of the number of legs could enhance tissue distension in collapsed cavities [22]. The main drawback of this approach is related to power supply and space constraints. The continuous use of a couple of actuators designed for moving two independent leg sets requires an energy source that may be hardly shrunk down to a swallowable size.

By combining external magnetic fields-which drag and orient the capsule-with a simplified internal active systemwhich creates space-a hybrid solution for controlled capsule endoscopy can be achieved. This technique would benefit from the specific advantages of both approaches and enable effective active locomotion in a swallowable size. The capsule described in this paper is a step toward this goal: it features a set of permanent magnets for external control in uncollapsed areas of the GI tract and a leg-based mechanism to be used as soon as the capsule gets stuck along its journey. This single leg set can additionally be exploited to lightly distend surrounding tissue and so obtain a reliable diagnostic outcome. Hybrid locomotion in capsule endoscopy is therefore firstly defined in this paper as the combination and synergy of magnetic and legged strategies for active control and locomotion in the LGI tract.

An overall description of the novel device is reported in Section II-A, followed by a detailed overview of the dimensioning of the internal (legged) and external (magnetic) locomotion mechanisms in Sections II-B and II-C, respectively. Control unit and power supply are discussed in Section II-D, while packaging issues are outlined in Section II-E. Section III describes the experimental assessment of the capsule, conducted to confirm technical requirements and performance of hybrid locomotion. Finally, conclusions and future improvements of the system are illustrated in Section IV.

\section{HYBRID CAPSULE DESIGN}

\section{A. System Overview}

The patient undergoing the devised procedure is requested to enter a purposely equipped ambulatory environment once the capsule has reached the LGI tract. Then, the endoscopist employs magnetic locomotion as much as possible [12] and guides the capsule using real-time vision feedback. Since natural gases are frequently present in the LGI tract, most of the capsule journey may occur by simple magnetic dragging, relying on the low dynamical friction coefficient of intestinal tissue, which is lubricated by a thick mucus layer [23]. If the capsule gets stuck in collapsed areas of the intestine, the on-board legged mechanism is activated and magnetic locomotion can take over again. Specifically, leg activation pushes the capsule outside a tissue fold if the capsule is entrapped. Alternatively, by keeping the legs open, the capsule can be driven by an oscillatory motion due to the magnetic link. This provides a lever-like action, which expands the tissue and allows the capsule to overcome sharp bends. Finally, the internal mechanism may also help dilate tissue and obtain a better view of intestinal walls. Fig. 1 shows an example of hybrid locomotion.

Starting from this operative scenario, it is possible to outline the desirable features that the hybrid capsule should have as follows.

1) Small size: The capsule should be small enough to swallow. However, since the hybrid locomotion concept still needs to be extensively validated, scalability of the prototype down to a swallowable size was considered acceptable in this paper. Thus, a diameter of $14 \mathrm{~mm}$ was assumed for this device, with a target of $11 \mathrm{~mm}$ for future developments.

2) Controllability by external magnetic fields in uncollapsed regions: Considering that the external source of the magnetic field is at an average operative distance of $10 \mathrm{~cm}$ from the capsule, the attraction force should be adequate to lift the capsule against its own weight. If this is satisfied, we can reasonably assume that the external magnetic field would also be able to drag the pill inside uncollapsed or lightly collapsed intestine.

3) Ability to expand surrounding tissues in collapsed regions: Previous studies have demonstrated that about $3 \mathrm{~N}$ of total force at leg tips are required to lift and stretch out collapsed tissue by a legged mechanism [10], [22].

4) Real-time vision system: Since the devised procedure is based on a real-time visual feedback from the capsule, a wireless vision module with adequate frame rate needed to be integrated. Despite research in this field [24], no miniaturized real-time vision system was available at the time of the work, therefore an estimated volume $\left(450 \mathrm{~mm}^{3}\right.$ as the ongoing efforts [25] toward a wireless vision module) for this module was left available in the hybrid capsule for future integration.

5) User-friendly control interface: Since capsule steering is obtained by applying two synergetic approaches, the endoscopist must be able to switch between the two easily and intuitively. 


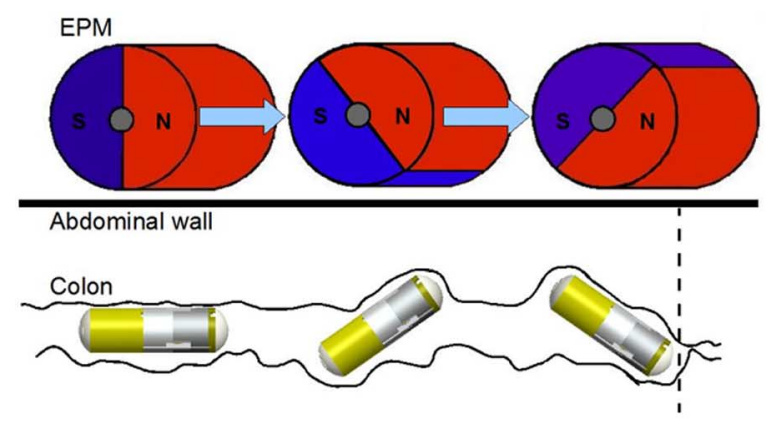

(a)

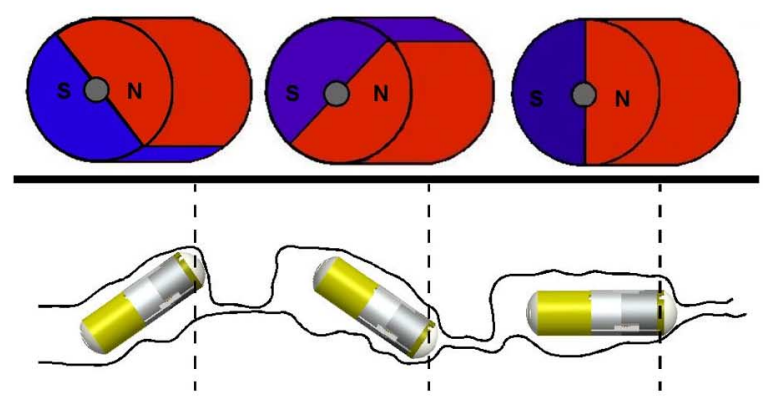

(b)

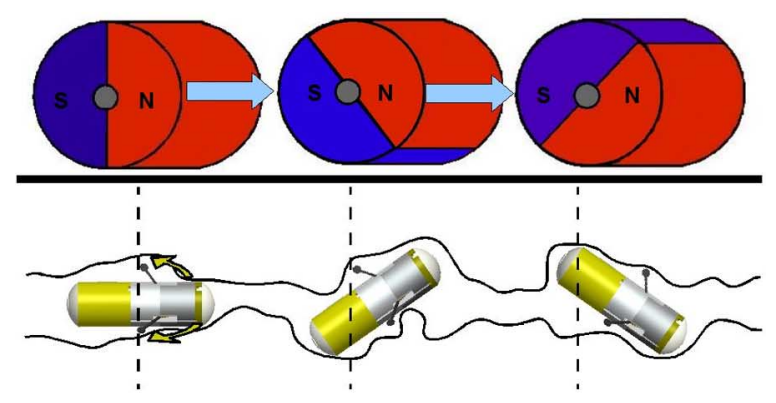

(c)

Fig. 1. (a) Legs are closed inside the body and the external magnet moves the capsule in lightly collapsed region. Collapsed tissue, corresponding to the dashed line, stops the magnetically driven motion. (b) External magnet only allows capsule orientation, and does not provide net locomotion. (c) Activation of the legged mechanism lifts the tissue and moves the capsule slightly forward. Magnetic locomotion starts again after removing the tissue around the capsule. The oscillating motion of the external magnet in combination with translation can help to overcome the sharp bends of the intestine.

\section{B. Internal Mechanism}

1) Legged System Design: The internal legged mechanism must be activated whenever the capsule is lodged within collapsed tissue or more lumen distension is required. To achieve this goal, a viable actuator was first selected, then the mechanism was designed and its parts were dimensioned considering the aforementioned requirements.

Due to previous extensive analysis regarding actuators for legged locomotion in capsule endoscopy [22], an electromagnetic brushless dc motor (SBL04-0829, Namiki Precision Jewel Company Ltd., Tokyo, Japan) was selected as the best commercially available tradeoff between output torque and compactness. The selected actuator had an external diameter of $4 \mathrm{~mm}$, a total length of $17.4 \mathrm{~mm}$ (not including the shaft), and a nominal

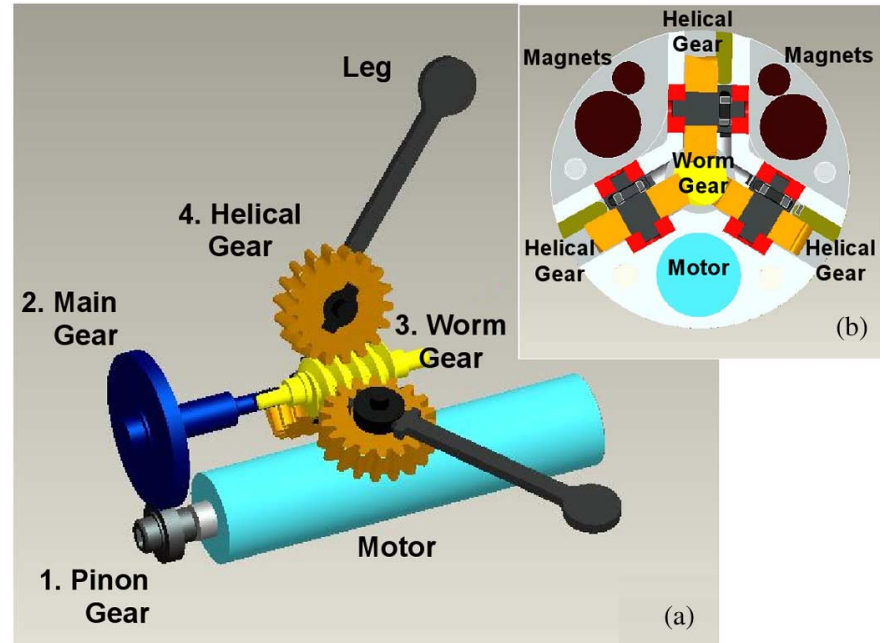

Fig. 2. (a) Schematic view of the hybrid capsule internal mechanism. Gears 1 and 2 do not have teeth in the picture for the sake of clarity. (b) Cross section view of the locomotion unit.

output stall torque of $5.7 \mathrm{mNm}$ [26]. Being an electromagnetic motor, permanent magnets placed close to the rotor or externally generated intense magnetic fields reduce effective output torque. As reported in the following sections, this issue was deeply analyzed for a proper dimensioning of the magnetic system. Alternative actuators, such as piezoelectric motors were also considered. Despite their immunity to magnetic fields, those commercially available do not guarantee a high torque in a small size, as desirable for this application.

As regards the mechanism, the use of three legs placed radially at $120^{\circ}$, was considered adequate for positioning both mechanism components and magnets, and to obtain an effective tissue engagement. The pivoting point of the three legs was placed in the middle of the locomotion unit, thus obtaining a symmetrical and reversible $180^{\circ}$ movement. Each leg snapped into a rectangular groove obtained on the lateral surface of a brass shaft, which was connected to a bronze helical gear [part 4 in Fig. 2(a)] by means of a custom-shaped groove.

A steel worm gear [part 3 in Fig. 2(a)] placed on the central axis of the locomotion unit matched the three helical gears, whereas a brass main gear [part 2 in Fig. 2(a)] transmitted the motion to the worm gear due to a long $(5.6 \mathrm{~mm})$ axial shaft. Finally, a brass pinion gear [part 1 in Fig. 2(a)] connected the mechanism to the dc brushless motor. As visible in the cross section view of Fig. 2(b), the mechanism design divides the capsule in three longitudinal sections. One section hosts the dc brushless motor, while the other two are left available for the on-board magnets.

2) Legged System Dimensioning: In order to define the design parameters for the different gears of the mechanism, several issues were considered, such as dc brushless motor features, capsule size, and ease of fabrication. A high reduction ratio is a key point for this application, because it allows the force available at the leg tip to be increased.

The relation between output $\left(M_{\text {out }}\right)$ and input $\left(M_{\text {mot }}\right)$ torques as function of total mechanism efficiency $\left(\eta_{\text {tot }}\right)$ and total 
transmission ratio $\left(\tau_{\text {tot }}\right)$ was as follows:

$$
M_{\mathrm{out}}=\frac{M_{\mathrm{mot}} \eta_{\mathrm{tot}}}{\tau_{\mathrm{tot}}}
$$

Considering the hybrid mechanism model, $M_{\text {out }}$ was the torque of both legs and respective helical gears, $M_{\text {mot }}$ was the torque transmitted by the motor to the mechanism, $\tau_{\text {tot }}$ was the total transmission ratio of the hybrid mechanism gears, whereas $\eta_{\text {tot }}$ was the total efficiency.

Considering the kinematic chain, $\tau_{\text {tot }}$ and $\eta_{\text {tot }}$ can be written as follows:

$$
\begin{aligned}
\tau_{\text {tot }} & =\tau_{12} \tau_{23} \tau_{34} \\
\eta_{\text {tot }} & =\eta_{12} \eta_{23} \eta_{34}
\end{aligned}
$$

where subscripts 1, 2, 3, 4 indicate the gears as in Fig. 2(a). The output torque can also be written as follows:

$$
M_{\text {out }}=F_{\text {legs }} b
$$

where $b$ was the lever arm defined by the leg length and $\mathrm{F}_{\text {legs }}$ was the total force at the leg tips.

The motor torque can be defined as follows:

$$
M_{\mathrm{mot}}=0.5 C_{\mathrm{mot}} \gamma_{\mathrm{mot}}
$$

where $C_{\mathrm{mot}}$ was the stall torque for the selected motor $\left(C_{\mathrm{mot}}=\right.$ $6.95 \mathrm{mN} \cdot \mathrm{m}$, when powered with $3.7 \mathrm{~V}$ ). In order to introduce a safety margin for the mechanism design, we adopted the half of this value as the operative motor torque. As previously mentioned, permanent magnets placed close to an electromagnetic motor reduce performance of the actuator. To consider this, we introduced an experimental coefficient $\left(\gamma_{\mathrm{mot}}\right)$ that related the loss of torque available for moving the mechanism to the magnetic field surrounding the motor.

From (1) and considering (2)-(5), the hybrid three-legged mechanism can be described by

$$
\begin{aligned}
F_{\text {legs }} b & =\frac{\left(0.5 C_{\mathrm{mot}} \gamma_{\mathrm{mot}}\right)\left(\eta_{12} \eta_{23} \eta_{34}\right)}{\tau_{12} \tau_{23} \tau_{34}} \Rightarrow \\
F_{\text {legs }} & =\frac{\left(0.5 C_{\mathrm{mot}} \gamma_{\mathrm{mot}}\right)\left(\eta_{12} \eta_{23} \eta_{34}\right)}{\left(\tau_{12} \tau_{23} \tau_{34}\right) b} \geq 3 \mathrm{~N}
\end{aligned}
$$

for an optimal management of collapsed tissue.

On the basis of (6) and the 4-leg capsule described in [16], the mechanism gears were designed to obtain the best tradeoff between maximum reduction ratio, high efficiency, small size, reliability, fabrication easiness, and costs, according to guidelines reported in [27] and [28]. The main features of gears and mechanism are summarized in Tables I and II, respectively.

The mechanical transmission between worm and helical gears allowed a very high reduction ratio $\left(\tau_{34}=0.056\right)$, a very compact size, and high reliability to be achieved. On the other hand, the high friction between the helical teeth hampered efficiency, resulting equal to $\eta_{34}=0.415$ as in [16].

Transmission between worm and main gears was designed with a fixed joint (also the connection between pinion gear and motor shaft) and defined unitary transmission ratio and efficiency $\left(\tau_{23}=1\right.$ and $\left.\eta_{23}=1\right)$.
TABLE I

GEAR FEATURES

\begin{tabular}{|l||l|l|l|l|}
\hline & $\begin{array}{l}\text { PINION } \\
\text { GEAR }\end{array}$ & $\begin{array}{l}\text { MAIN } \\
\text { GEAR }\end{array}$ & $\begin{array}{l}\text { WORM } \\
\text { GEAR }\end{array}$ & $\begin{array}{l}\text { HELICAL } \\
\text { GEARS }\end{array}$ \\
\hline \hline MODULUS & 0.15 & 0.15 & 0.25 & 0.25 \\
\hline $\begin{array}{l}\text { TEETH NUM- } \\
\text { BER }\end{array}$ & 16 & 44 & 1 & 18 \\
\hline $\begin{array}{l}\text { PITCH } \\
\text { CIRCLE }\end{array}$ & $2.4 \mathrm{~mm}$ & $6.6 \mathrm{~mm}$ & $2.051 \mathrm{~mm}$ & $4.534 \mathrm{~mm}$ \\
\hline ADDENDUM & $0.15 \mathrm{~mm}$ & $0.15 \mathrm{~mm}$ & $0.25 \mathrm{~mm}$ & $0.25 \mathrm{~mm}$ \\
\hline DEDENDUM & $0.1875 \mathrm{~mm}$ & $0.1875 \mathrm{~mm}$ & $0.3125 \mathrm{~mm}$ & $0.3125 \mathrm{~mm}$ \\
\hline $\begin{array}{l}\text { PRESSURE } \\
\text { ANGLE }\end{array}$ & $20^{\circ}$ & $20^{\circ}$ & $20^{\circ}$ & $20^{\circ}$ \\
\hline $\begin{array}{l}\text { HELIX } \\
\text { ANGLE }\end{array}$ & - & - & $7^{\circ}$ & $7^{\circ}$ \\
\hline
\end{tabular}

TABLE II

MECHANISM FEATURES

\begin{tabular}{|l||l||l|}
\hline & $\begin{array}{l}\text { Transmission } \\
\text { ratio }\end{array}$ & Efficiency \\
\hline \hline PINION GEAR-MAIN GEAR & $\tau_{12}=0.364$ & $\eta_{12}=0.9$ \\
\hline MAIN GEAR-WORM GEAR & $\tau_{23}=1$ & $\eta_{23}=1$ \\
\hline WORM GEAR-HELICAL GEAR & $\tau_{34}=0.056$ & $\eta_{34}=0.415$ \\
\hline
\end{tabular}

A low modulus (equal to 0.15) was selected for both the pinion and the main gears, thus obtaining homogeneous rotation. Considering the relation in [28], these two gears also featured a transmission ratio of $\tau_{12}=0.364$ and an efficiency of $\eta_{12}=$ 0.9 .

From the gear features, it is possible to calculate total mechanism transmission ratio and total efficiency $\left(\tau_{\text {tot }}=0.02\right.$ and $\eta_{\text {tot }}=0.3735$ ).

Both pinion and main gears were fabricated by using a five-axis micro-CNC machining center (HSPC, KERN GmbH, Germany). The steel worm gear and the bronze helical gears were custom designed by the authors and fabricated by an external workshop. The gears were modified by sink and microwire electrodischarge machining (EDM) (Micro Sink, Sarix, Switzerland, and AP 200L, Sodick, Japan, respectively) to provide proper couplings between connecting parts. In particular, the worm gear was cut at one end in order to obtain a rectangular groove, while a T-shaped hole was made in each helical gear to enable proper connection with the brass shaft. The three brass shafts were fabricated by the $\mathrm{CNC}$ machining center. The motor shaft was machined by microwire EDM and fitted into the hole previously obtained in the center of the pinion gear. Finally, the gears were assembled on custom made bushes in synthetic ruby with buffing surface minimizing friction force and overall dimensions. The fabricated components of the legged mechanism are showed in Fig. 3.

After dimensioning and fabricating the gears, the legs were designed in terms of shape and length, on the basis of (6) and size requirements. Since the legs needed to lift tissues rather than to propel, the leg movement was conceived as reversible 


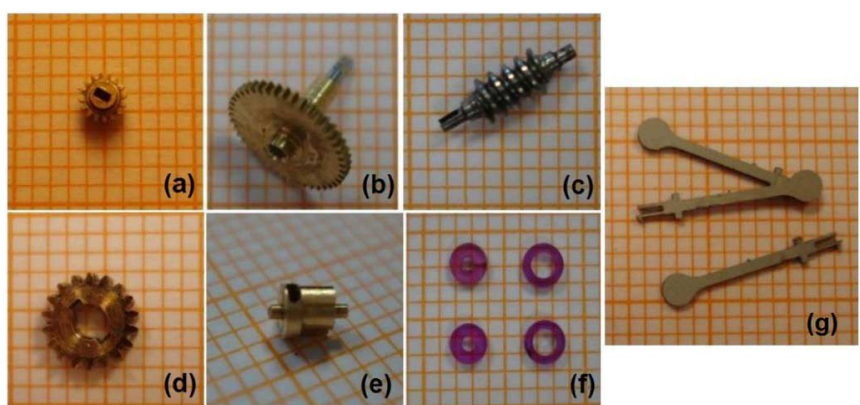

Fig. 3. Internal components of the legged mechanism. (a) Pinion gear. (b) Main gear. (c) Worm gear. (d) Helical gear. (e) Brass shaft. (f) Bushes. (g) NiTinol legs. All components are depicted on millimetric sheets.

(moving forward and backward, and backward and forward). Therefore, a simple symmetrical leg geometry was selected. A round shape was adopted for the leg tip in order to minimize local tissue stresses. The legs were fabricated from a $0.5 \mathrm{~mm}$ thickness superelastic alloy (NiTinol) plate by microwire EDM [see Fig. 3(g)]. Superelastic alloy was chosen because it provides high elasticity, particularly useful at the leg-shaft junction [29].

The total length of the locomotion module was $21 \mathrm{~mm}$, while the legs were $10.5 \mathrm{~mm}$ long and could be symmetrically closed on both sides. Finally, considering (6) and assuming the force requirement $F_{\text {legs }}$ as $3 \mathrm{~N}$, it was possible to calculate the minimum value for $\gamma_{\text {mot }}$, which still met our specifications as 0.5 .

The following paragraph describes the dimensioning of magnetic system, which led to the determination of $35 \%$ performance loss $\left(\gamma_{\text {mot }}=0.65\right)$, this corresponds to $F_{\text {legs }}=3.8 \mathrm{~N}$, thus providing a safety margin on the desired specifications. If extensive tests were to demonstrate that a lower motor performance is sufficient, the force requirements could be reduced, as also the size of the entire locomotion module.

\section{Magnetic System}

The magnetic system provides external active locomotion in partially collapsed or uncollapsed regions of the LGI tract by coupling of external and internal magnetic fields.

To help endoscopists easily handle the external source of magnetic field, a cylindrical $\mathrm{NdFeB} \mathrm{N} 35$ permanent magnet placed on a passive hydraulic arm, was adopted (see Fig. 4). Once the hybrid locomotion has been assessed, a robotic arm may be used to replace the passive support, as proposed in [15].

The main features of the external permanent magnet (EPM) were: a cylindrical shape $(\phi=60 \mathrm{~mm}, \mathrm{~L}=70 \mathrm{~mm})$ with an axial hole $(\phi=10 \mathrm{~mm})$ to allow fixation, a $1.5 \mathrm{~kg}$ weight, a magnetic remanence of $1.21 \mathrm{~T}$ due to the selected material, and a magnetization vector perpendicular to the main axis of the cylinder.

First finite-elements method (FEM) analysis was performed with COMSOL Multiphysics 3.4 (COMSOL Inc., Sweden) with the aim to obtain the relationship between magnetic field and distance from the surface of the EPM. All the EPM properties, such as shape, dimension, magnetization direction, remanence, and magnetic permeability $\left(\mu_{0}=1.05\right)$ were set into the simulation. The selected mesh consisted in about 450.000 elements

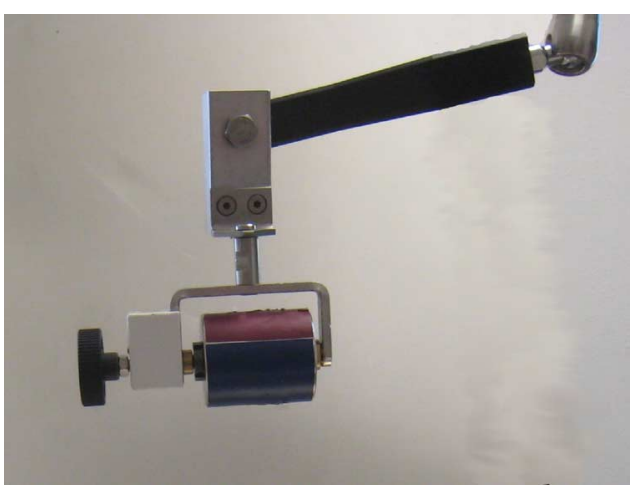

Fig. 4. EPM mounted on the hydraulic arm.

with a maximum element size set at 1/50 of the maximum geometric feature in the scenario. The incremental ratio of the mesh elements was set to 1.3 , while the curvature factor and the mesh curvature cutoff were 0.2 and 0.001 , respectively.

In order to quantify $\gamma_{\mathrm{mot}}$, we assumed that the effect of the magnetic field acts as a torque working against the motor torque itself. To verify this assumption, we performed the following experimental procedure: firstly, given a fixed voltage supply $(3.7 \mathrm{~V})$, motor rotational speed, and the related current absorption were measured as a function of distance between the motor and the EPM surface. Then, considering the motor characteristic curves, relating shaft rotational speed, current absorption, and motor torque [26], we confirmed that rotational speed and current absorption behave as if an increasing load was applied to the shaft when the EPM-motor distance decreased. This allowed us to assume the effect of the magnetic field surrounding the motor as an external antagonistic torque $\left(C_{\mathrm{mag}}\right)$ exerted on the motor shaft. This perturbation reduced the torque $\left(M_{\mathrm{mot}}\right)$ available for moving the mechanism.

The FEM analysis and the experimental results were used to obtain a plot describing the experimental trend of the motor rotational speed and the current absorption, both as a function of magnetic flux density. The rotational speed of the shaft decreased with magnetic field, whereas the current absorption increased. The torque $C_{\mathrm{mag}}$ was derived form the motor characteristic curves and the measures of speed and current. Since rising with the magnetic field, the coefficient $\gamma_{\text {mot }}$ described by the (7) decreased, as shown in Fig. 5

$$
\gamma_{\mathrm{mot}}=\frac{0.5 C_{\mathrm{mot}}-C_{\mathrm{mag}}}{0.5 C_{\mathrm{mot}}} .
$$

The internal magnetic field was generated by a set of small permanent magnets [internal permanent magnets (IPM)] embedded into the capsule. The optimal IPM configuration would maximize the volume of magnets into the space left available inside the locomotion unit (roughly $500 \mathrm{~mm}^{3}$ ). It would also minimize motor interferences $\left(\gamma_{\mathrm{mot}}=0.65\right)$ and maximize magnetic attraction force with the selected EPM, according to the requirements presented in Section II-A.

Off-the-shelf cylindrical NdFeB N50 permanent magnets, having a $1.45 \mathrm{~T}$ magnetic remanence were selected as IPM with the purpose of maximizing the magnetic flux density for a 


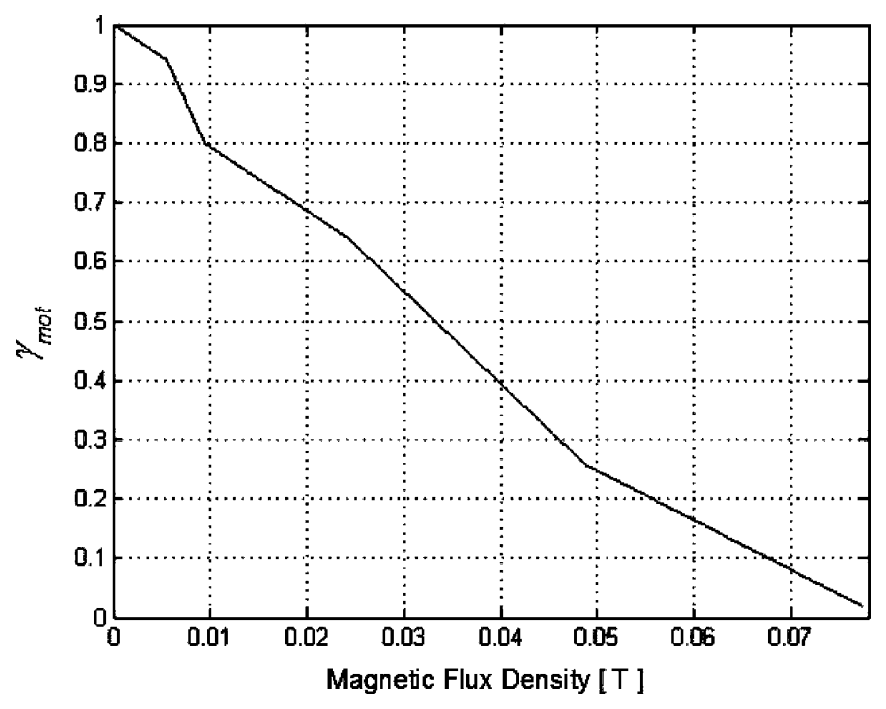

Fig. 5. Experimental relationship between $\gamma_{\mathrm{mot}}$ and magnetic flux density.

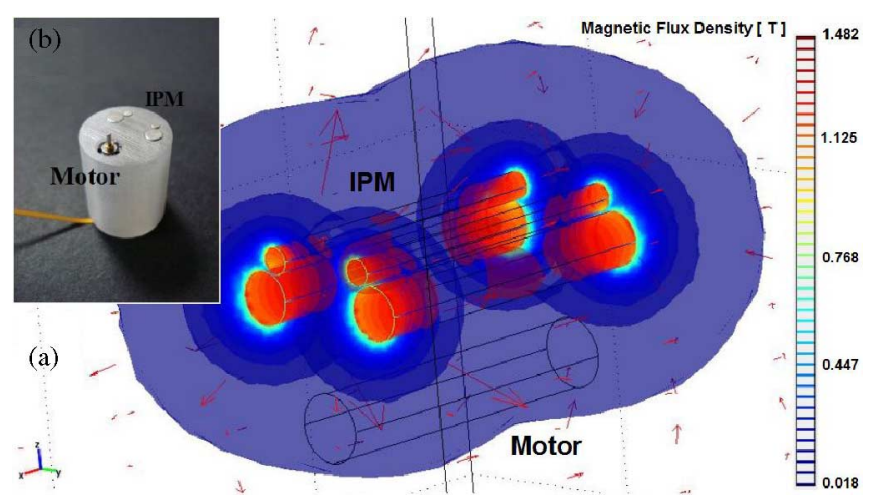

Fig. 6. (a) FEM simulation of the selected IPM configuration: plot of magnetic flux density. (b) Experimental test bench to quantify $\gamma_{\mathrm{mot}}$.

given volume. Given the shape and the volume (i.e., $500 \mathrm{~mm}^{3}$ ) of the space available inside the capsule, as also our previous experience in magnetic steering of endoscopic capsule [15], the ten most suitable IPM configurations, in terms of number and arrangement of the magnets composing the IPM, were simulated. The main goal was to obtain a rapid solution for each configuration in terms of magnetic field engaging the motor and magnetic attraction force with the EPM. A complete analytical solution for the forces between permanent magnets was based on Kelvin's formula and on Biot-Savart's law as reported in [30].

The selected IPM configuration [see Fig. 6(a)] was composed by six cylindrical NdFeB N50 magnets $(\phi=3.175 \mathrm{~mm}, L=$ $6.35 \mathrm{~mm})$ and 12 cylindrical NdFeB N50 magnets $(\phi=$ $1.58 \mathrm{~mm}, L=3.175 \mathrm{~mm}$ ) with axial magnetization and total volume of $475 \mathrm{~mm}^{3}$ (KJ Magnetics, Jamison). FEM analysis of this configuration showed that the motor was engaged in an average magnetic field of $25 \mathrm{mT}$. Thus, the parameter $\gamma_{\text {mot }}$ was estimated as 0.65 (see Fig. 5), meeting the requirements. The simulated magnetic attraction between IPM and EPM [see Fig. 7(a)] was calculated as $135 \mathrm{mN}$ at $10 \mathrm{~cm}$ and $550 \mathrm{mN}$ at $5 \mathrm{~cm}$, enabling the capsule (approximately $13.5 \mathrm{~g}$ ) to be lifted within this distance range. Another outcome of this simulation

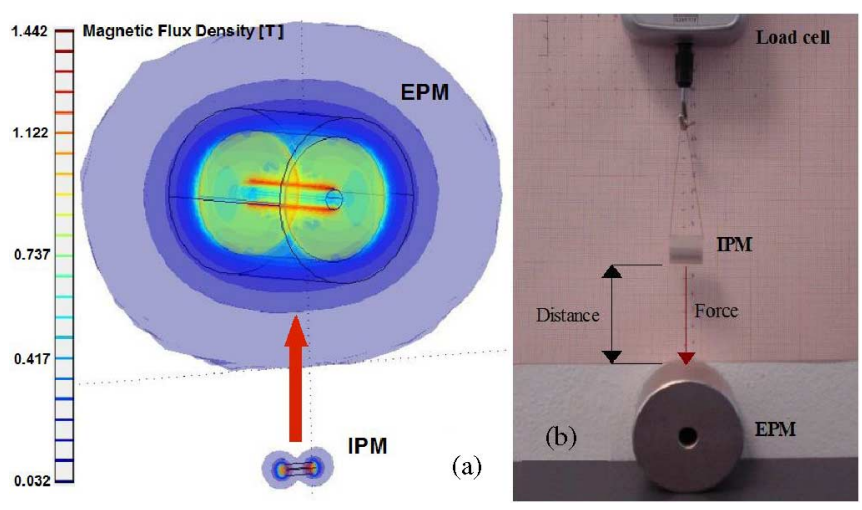

Fig. 7. (a) FEM simulation to evaluate magnetic attraction force between EPM and IPM. (b) Experimental test bench with the IPM connected to a load cell.

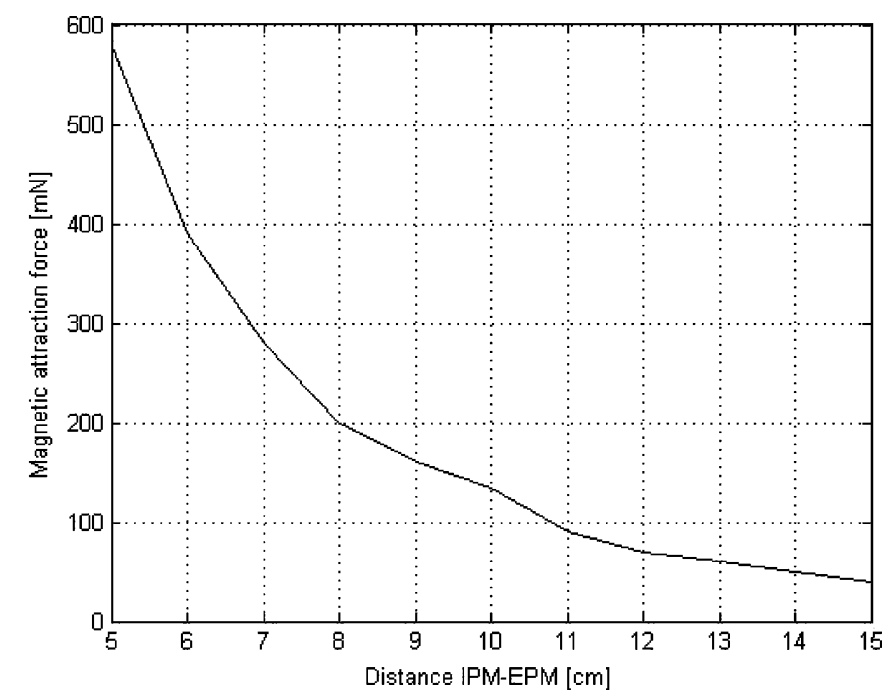

Fig. 8. Experimental plot of magnetic attraction force versus EPM-IPM distance in the selected configuration.

was the negligible effect of the EPM magnetic field on the motor performance at the operative distance of $10 \mathrm{~cm}$. The force requirement of $3 \mathrm{~N}$ defined in Section II-A, was still fulfilled at an EPM-capsule distance of $9 \mathrm{~cm}\left(\gamma_{\mathrm{mot}}=0.5\right)$, while at shorter distances the interference increased until the mechanism stopped at $5 \mathrm{~cm}$. In this case, only magnetic locomotion could be used. It is worth noting that in the case of obese patients, a $10 \mathrm{~cm}$ distance between EPM and IPM may not be a feasible option. In this case, a different EPM needs to be used, either by increasing its volume or selecting a material with higher remanence.

In order to verify FEM results, experimental tests were performed with the selected configuration. Firstly, a $\gamma_{\text {mot }}$ of 0.65 was experimentally assessed with a dedicated test bench reported in Fig. 6(b). Then, a monoaxial load cell (FMI-210, Alluris, Germany) was used to verify the attraction force between EPM and IPM at different distances [see Fig. 7(b)]. The force measured with the load cell plotted in Fig. 8, confirmed the FEM analysis results. 


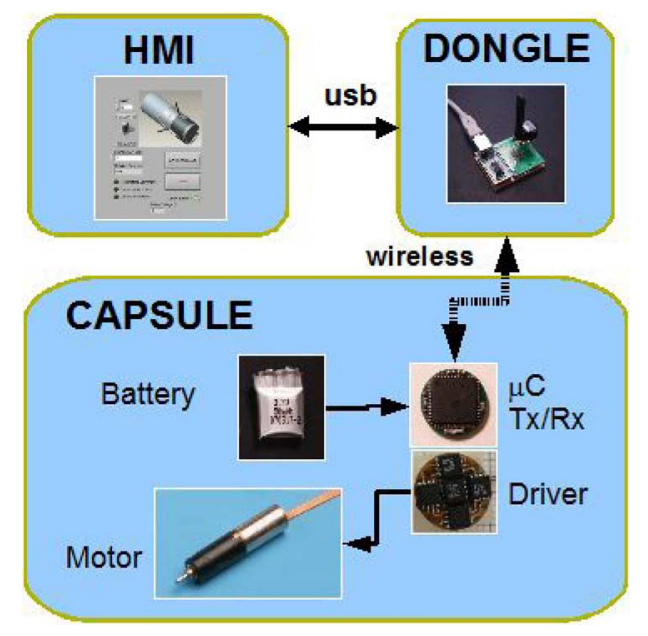

Fig. 9. Communication architecture.

\section{On-Board Electronics}

The hybrid capsule integrated a four-layer circular electronic board $(\phi=10.3 \mathrm{~mm}$, thickness $=2.5 \mathrm{~mm})$ with a wireless 8051 microcontroller (CC2430, Texas Instruments) and a driver for brushless dc motor activation [31]. The microcontroller embedded an IEEE 802.15.4 compliant wireless transceiver working at $2.4 \mathrm{GHz}$. This proved to be an effective solution for LGI tract telemetry, as reported in [32]. The capsule was interfaced with a standard PC through a custom designed universal serial bus (USB) dongle, featuring a second CC2430 and a serial USB converter (UM232, FDTI Chip, U.K.). A purposely developed human-machine interface (HMI) ran on the PC, enabling the user to control the capsule and receive a feedback on its status (see Fig. 9). The HMI was implemented with labview (National Instruments) and it was also compatible with voice commands. The endoscopist, therefore, by pronouncing the proper commands (including direction and angle) could open the legs while operating the magnet.

Deep sleep modality was also implemented, thus allowing the capsule to use just $0.3 \mu \mathrm{A}$ when in idle mode. Two external contacts if short-circuited, enabled the system to wake up and start communication with the HMI.

As regards the energy source, a single rechargeable lithium ion polymer battery (LP50, Plantraco, Canada) was used to maximize the ratio between capacity and volume. Its main features were: peak current of $500 \mathrm{~mA}, 3.7 \mathrm{~V}$ voltage, compact size $\left(9.5 \times 11 \times 3.3 \mathrm{~mm}^{3}\right)$, and a $50 \mathrm{mAh}$ nominal capacity. The battery only needs to be charged during the development phase, since multiple tests may be conduced without the need to replace the energy source.

In order to estimate the battery lifetime, current absorption in different working modalities was measured with a digital oscilloscope. Average current drain during periodic activation of wireless transmission was $2.75 \mathrm{~mA}$, whereas motor activation required an average current of $120.6 \mathrm{~mA}$. This resulted in a range going from $18 \mathrm{~h}$ (only telemetry activities) to $25 \mathrm{~min}$ (continuative motor activation) depending on the use of the legged mechanism. Considering that the legs requires $16.7 \mathrm{~s}$

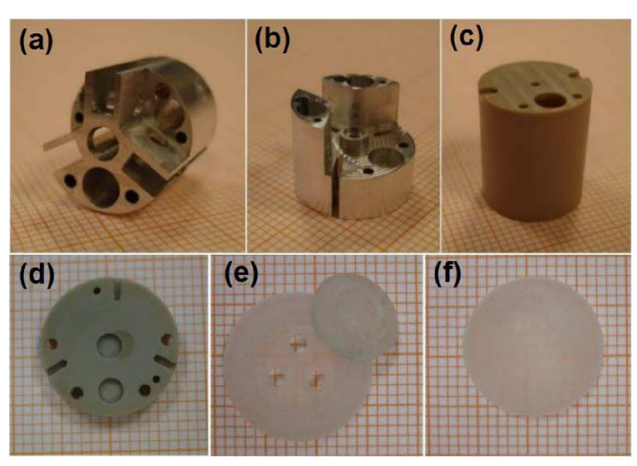

Fig. 10. (a) Chassis. (b) Body. (c) Tank. (d) Frontal plug. (e) Rear plug. (f) Cap.

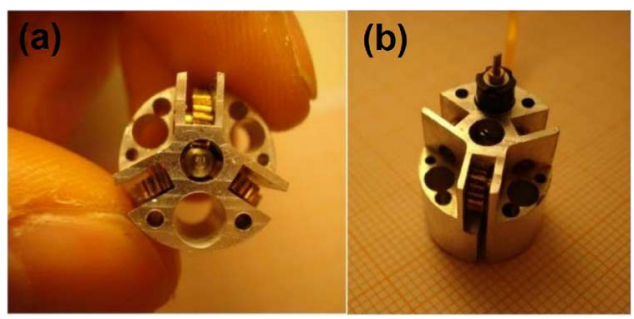

Fig. 11. (a) Assembly of worm gear and helical gears in the chassis. (b) Assembly of the motor.

to complete a $180^{\circ}$ span, 90 cycles can be performed by the capsule before the battery expires. From a different perspective, each $180^{\circ}$ leg movement consumes about $12 \mathrm{~min}$ of device autonomy.

\section{E. Packaging and Assembly}

After defining all the main features of the capsule, a compact packaging was designed, which focused on small overall dimensions, robustness, and ease of assembly. Biocompatibility of materials was not addressed at this stage of development and will be pursued, once the hybrid capsule will be completely assessed.

The legged mechanism was included in two complementary parts, named chassis and body, as in Fig. 10 that can be joined together by five screws. This solution ensured stability of the mechanical components and correct protection of the electrical parts. Chassis and body were fabricated in Ergal (i.e., an aluminum alloy) by CNC machining. Assembly of the legged mechanism components in the chassis is illustrated in Fig. 11.

A cylindrical tank [see Fig. 10(c)] was fabricated in polyarylether ether ketone (PEEK), a thermoplastic semicrystalline polymer, which hosted both electronics and battery. Additional parts (frontal and rear plugs, [Fig. 10(d)-(e)]) were fabricated either in PEEK or in acrylic photopolymer to match the single parts of the capsule and to make the device waterproof.

Three holes in the rear plug contained three magnetic contacts that wake up the capsule from deep sleep and recharge the battery. A hemispherical cap [see Fig. 10(f)], simulating the presence of a vision system, was fabricated with a stereolithographic technique (3-D Printer Invision Si2) and fixed frontally. 


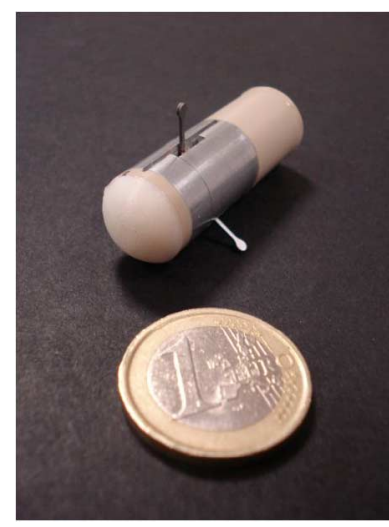

Fig. 12. First hybrid capsule prototype.

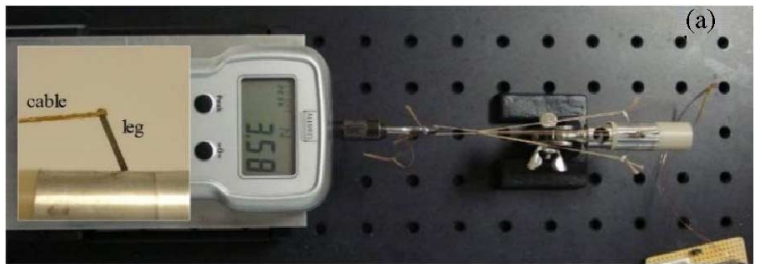

(b)

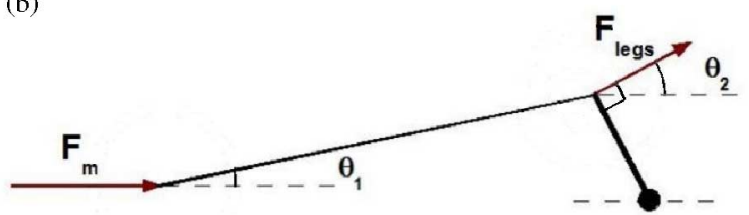

Fig. 13. Leg force measurements. (a) Cable is fixed at the tip of each leg. The load cell measures the tension of the cable. (b) Simple model for calculating effective leg force.

Fig. 12 shows the wireless hybrid capsule prototype. It is composed roughly of 60 different parts, and once assembled, measures $14 \mathrm{~mm}$ in diameter, $44 \mathrm{~mm}$ in length, and weights $13.5 \mathrm{~g}$.

\section{EXPERIMENTAL VALIDATION}

\section{A. Bench Testing}

The first experiments were performed to assess if the mechanical features of the prototype met the design specifications. In order to measure the force generated by the three legs, three nonextensible cables of the same length were fixed on the tip of each leg. The cables were then tied together and fixed to a monoaxial load cell, as in Fig. 13(a). When the motor was activated by a wireless command, the legs pulled the cables and an average force of $3.58 \mathrm{~N}$ was acquired by the load cell. By measuring the cable and leg angles $\left(\theta_{1}=8^{\circ}\right.$ and $\left.\theta_{2}=28^{\circ}\right)$, as defined in Fig. 13(b), we derived the $F_{\text {legs }}$.

By using a simple vectorial sum

$$
F_{\text {legs }}=\frac{F_{m}}{\cos \left(\theta_{1}\right) \cos \left(\theta_{2}-\theta_{1}\right)}
$$

it was possible to calculate the effective force generated by the three legs as $3.85 \mathrm{~N}$. This value met the mechanical requirements listed in Section II-A.

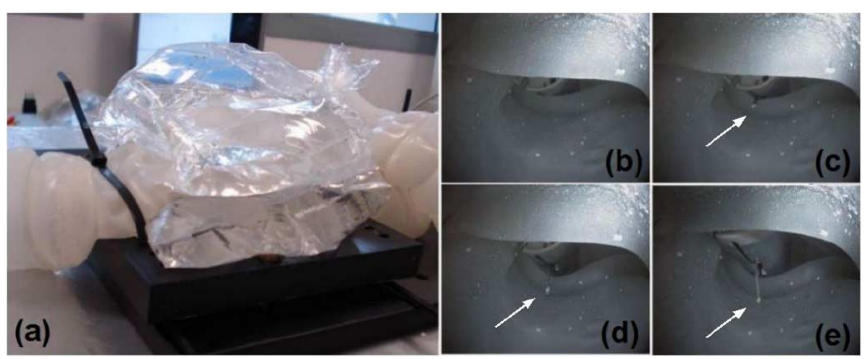

Fig. 14. (a) In vitro bench test. (b) Capsule is stuck in the cavity. (c)-(e) Activation of the legged mechanism to get rid of collapsed lumen (see white arrow).

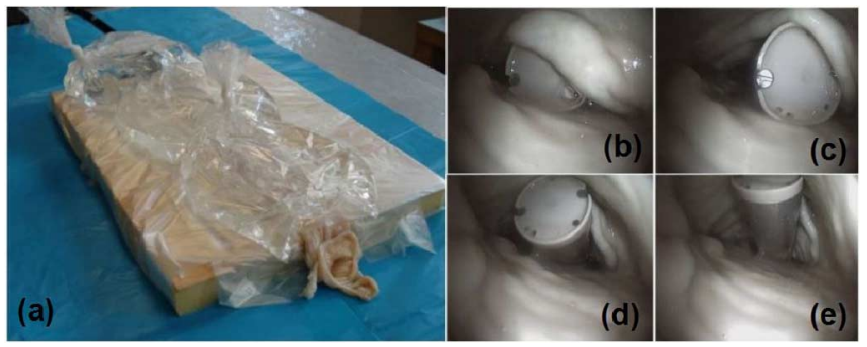

Fig. 15. (a) Ex vivo bench test. (b)-(d) Activation of the legged mechanism to get rid of collapsed tissue. (e) Magnetic locomotion starts again after removing the tissue around the capsule.

\section{B. Locomotion Performance Assessment}

Setting up a proper test bench and a reliable protocol to assess hybrid capsule performance is no trivial matter, since uncollapsed regions in the human LGI are not easily predictable in terms of number and extension. We implemented preliminary in vitro, ex vivo, and in vivo trials to estimate average transit time of the capsule provided with active locomotion. However, an extensive statistical analysis of a relevant number of trials must be performed in order to obtain a definitive evaluation of the proposed device. Throughout the experiments, the capsule was observed by a gastroscope (Karl Storz Endoscopy, Tüttlingen, Germany) in order to verify capsule motion and to assess the lumen dilatation generated by the three legs.

Ten preliminary in vitro tests were conducted in a $20 \mathrm{~cm}$ tract of latex colon simulator (Kyoto Kagaku, Kyoto, Japan). Then, ten ex vivo trials with a $20 \mathrm{~cm}$ fresh porcine colon segment were performed. Considering the weight of the abdominal wall on the colon [33] and the force required for the dilatation of a collapsed tract [10], [22], the intestine was covered with water filled plastic bags (about $350 \mathrm{~g}$ each) [see Figs. 14(a) and 15(a)] in order to simulate the surrounding tissues.

The EPM was placed on the hydraulic arm and maintained at an operative distance of $10 \mathrm{~cm}$ from the capsule during all tests. The capsule was dragged by the EPM as much as possible and the legged mechanism was activated only when the capsule was stuck in tissue folds. Based on the time required to complete a traditional colonoscopic procedure [34], the trial was considered failed if the capsule was not able to cross the entire segment after $10 \mathrm{~min}$.

Both in vitro and ex vivo bench tests confirmed that the magnetic field coupling can move and steer the capsule in slightly 
collapsed regions, whereas activation of the legged mechanism (especially when moving forward, and then, backward) was able to lift and distend collapsed tissues or to overcome tissue folds.

In all in vitro tests the capsule reached the end of the simulator within the 10 min time frame, whereas in the ex vivo trials it was able to travel along the intestine tract in only seven out of ten cases. The latex colon simulator had an averagely larger lumen (about $\phi=35 \mathrm{~mm}$ ) than the ex vivo specimen and it also tended to hold the original shape of the cavity despite the weight of the bags, thus facilitating magnetic locomotion. Although the ex vivo colon specimen was very slippery and was positioned on the bench without bends, the three failed trials were mainly due to the absence of muscular tone (as in the in vivo condition), which resulted in completely collapsed tissue around the capsule. It is worth mentioning that failure does not mean capsule malfunctioning, but inability to cover the entire $20 \mathrm{~cm}$ of the specimen in the targeted time frame.

The capsule needed an average time of 2 min and two legged mechanism activations to go through the latex colon simulator about $4 \mathrm{~min}$ and five mechanism activations were required in the porcine lumen (excluding the three failed trials). The average speed was estimated as 10 and $5 \mathrm{~cm} / \mathrm{min}$ for in vitro and ex vivo tests, respectively.

Ten in vivo trials were performed on a total of four female pigs with a weight ranging from 25 to $35 \mathrm{~kg}$. The experiments were conducted in an authorized laboratory with the assistance and collaboration of a specially trained medical team, in compliance with all ethical requirements and regulatory issues related to animal experiments. After intravenous sedation of the animal and preparation of the bowel with water enemas, an endoscope was used to introduce the capsule and position it $40 \mathrm{~cm}$ from the anus. The legs of the capsule were closed into the body in order to facilitate sliding. Then, while the gastroscope observed the capsule without insufflation, the hybrid device was moved and steered by means of magnetic forces in order to simulate a diagnostic procedure. Magnetic locomotion always allowed precise orientation of the device, but the motion was only possible in uncollapsed or slightly collapsed regions. During the in vivo tests, the capsule was often lodged because either the tissues surrounded it completely or the cavity in front of it was highly collapsed. Activation of the legged mechanism enabled the layout of the capsule to be modified and the lumen to be enlarged. The motion of the three legs, moving from front to back, lifted the tissue easily and propelled the capsule forward. Experimental evidence showed that partial opening (around $45^{\circ}$ ) of the legs on the rear side was the best capsule layout for magnetically assisted locomotion, since the tissue was kept away from the device surface. With this configuration, a leverage effect of the legs on the collapsed tissue was achieved by oscillating the EPM, and sharp bends were effectively handled. Finally, legs motion from back to front allowed the capsule to move backward. This was beneficial whenever the capsule was stuck in a tissue pocket, such as a diverticulum. Naturally, the legs could be closed either toward the front or the back, however, they could not be closed a priori in order to impart the most favorable motion to the capsule when approaching an obstacle. Nevertheless, by correctly manoeuvering the EPM, a $135^{\circ}$ mo-

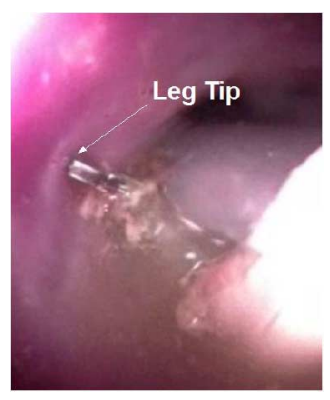

Fig. 16. Capsule opens its legs while immersed in physiological fluids.

tion can be obtained without tissue entrapment. At this point, the legs could be ready to move in the most favorable direction.

Ten locomotion trials were performed during the in vivo experimental sessions. In six cases, the capsule covered the entire $40 \mathrm{~cm}$ tract toward the anus in an average time of $5 \mathrm{~min}$ and an average speed of $8 \mathrm{~cm} / \mathrm{min}$. An average number of five legged mechanism activations was required. However, in four trials, the capsule was not able to complete the journey within the $20 \mathrm{~min}$ time frame. This was mainly due to the geometry of the porcine colon, which is much more complex than in humans. Understanding the correct leg activation sequence, also contributed to determining the high number of failures (three out of four failures occurred during the first half of the trials).

During the in vivo tests, the capsule was also moved against natural peristalsis, showing satisfactory performance. It is also worth mentioning that the hybrid capsule engaged in physiological fluids, as represented in Fig. 16, without any significant loss in performance, thus demonstrating its reliability and robustness.

\section{CONCLUSION}

The hybrid capsule developed, fabricated, and tested by the authors represents an effective solution for the active locomotion of endocapsules in the GI tract. The capsule incorporates several innovative features in terms of combination of different actuation principles. In fact, two different solutions for active locomotion (i.e., external magnetic actuation and internal actuation structures) were merged into the same device to overcome the most significant limitations of the two separate approaches. Combining permanent magnets and electromagnetic actuators is not a trivial matter due to interference that reduces the motor torque needed to move the mechanism. The paper contributes to the design of miniaturized devices combining magnetic and dc motor actuation, and provides quantitative guidelines for merging the two principles.

The fabricated hybrid locomotion capsule is a wireless system conceived with a "totally on-board" philosophy: it embeds an internal motor mechanism, several permanent magnets, a wireless microcontroller, and a rechargeable battery. Once assembled, it measures $14 \mathrm{~mm}$ diameter, $44 \mathrm{~mm}$ in length, and weights $13.5 \mathrm{~g}$. Experimental bench tests reveled that the internal mechanism produces $3.85 \mathrm{~N}$ force at the leg tip and that magnetic field coupling between internal and external magnets 
provides an attraction force capable of performing controlled locomotion at an operative distance of $10 \mathrm{~cm}$.

Comparison with other locomotion modules designed for endoscopic capsules presented in literature can be conducted on the basis of several parameters. The most significant in our opinion, is power consumption. From this standpoint, the hybrid capsule combines the zero power required by magnetic dragging (which becomes ineffective in collapsed regions of the LGI tract), with the specific advantages that a leg set can provide (e.g., tissue dilatation and freeing of the capsule from tissue folds). Our most advanced legged capsule prototype [22] requires a $10 \mathrm{~mm}$ diameter and $30 \mathrm{~mm}$ long battery to complete colon examination.

Another significant parameter considered when comparing locomotion modules is speed. With special reference to our previous legged capsule prototypes, the 4-leg capsule [16] speed was $3 \mathrm{~cm} / \mathrm{min}$, the 8-leg capsule [21] speed was $4 \mathrm{~cm} / \mathrm{min}$, and the 12-leg capsule [22] speed was $5 \mathrm{~cm} / \mathrm{min}$, while the hybrid capsule achieved a speed of $8 \mathrm{~cm} / \mathrm{min}$ in the in vivo trials.

The results obtained with this first prototype support the feasibility of the hybrid locomotion concept. The next generation of hybrid capsules could be reduced to a swallowable size by further optimizing each component. Considering that the $3.85 \mathrm{~N}$ force produced by the internal structure seems to be grater than the force required to lift collapsed tissue, a miniaturized mechanism may be designed as next step. Smaller integrated motors could reduce the size of the capsule, whereas many other features could be modified to improve functionality. New leg shapes (e.g., with a smoother profile) could be studied to minimize local tissue stresses or to facilitate colon distension.

In order to enable actively controlled endoscopic procedures, a real-time wireless vision module with a high frame rate must be integrated on-board the capsule. Although a system with these features is not commercially available, on-going efforts toward this goal are provided in literature [24]. In particular, our group is developing a $20 \mathrm{fps}$ real-time wireless camera system for capsule endoscopy, weighing $3.5 \mathrm{~g}$ and measuring $450 \mathrm{~mm}^{3}$ in volume [25]. The system requires approximately $100 \mathrm{~mW}$ to operate, inclusive of four white LED illumination system. Once this module will be available for integration, the whole design process of the magnetic link will be reiterated, following the procedure described in this paper and taking into account the modified weight of the capsule. As regards power supply, the additional burden introduced by the vision subsystem can be powered by replacing the battery with a wireless power supply solution, as reported in [35].

While real-time image stream should be the main feedback for the operator, additional sensing strategies may be implemented in order to attain fully autonomous hybrid locomotion. In such a scenario, the EPM could be mounted as end-effector on a robotic arm, as in [15], and real-time localization of the capsule (based on magnetic fields [36]) would allow the system to autonomously run the legged mechanism whenever the capsule does not follow the EPM movement. Additionally, contact or pressure sensors on the surface of the capsule could be exploited in order to autonomously trigger activation of the legs.

\section{ACKNOWLEDGMENT}

The authors would like to thank N. Funaro and the Staff of the Center for Research in Microengineering Laboratory mechanical workshop for manufacturing the prototypes. They are also grateful to Dr. Trivella, Dr. Burchielli, and the Staff of the Experimental Surgery Center of San Piero a Grado (Pisa) for their help during the in vivo testing phase of the device. Finally, they would like to thank Dr. Piccigallo for his technical support on brushless motor analysis.

\section{REFERENCES}

[1] G. Iddan, G. Meron, A. Glukhovsky, and P. Swain, "Wireless capsule endoscopy," Nature, vol. 405, no. 6785, pp. 417-418, May 2000.

[2] M. Waterman and R. Eliakim, "Capsule enteroscopy of the small intestine," Abdom. Imaging, vol. 34, pp. 452-458, 2008.

[3] A. Moglia, A. Menciassi, P. Dario, and A. Cuschieri, "Clinical update: Endoscopy for small-bowel tumours," Lancet, vol. 14, no. 370, pp. 114 116, 2007.

[4] Y. C. Metzger, S. N. Adler, A. B. Shitrit, B. Koslowsky, and I. Bjarnason, "Comparison of a new PillCam SB2 video capsule versus the standard PillCam SB for detection of small bowel disease," Rep. Med. Imaging, vol. 2, pp. 7-11, 2009.

[5] Olympus Endocapsule. (2010, Feb.). [Online]. Available: www.olympuseuropa.com/endoscopy/

[6] S. Bang, J. Park, S. Jeong, Y. H. Kim, H. B. Shim, T. S. Kim, D. H. Lee, and S. Y. Song, "First clinical trial of the miro capsule endoscope by using a novel transmission technology: Electric-field propagation," Gastrointest. Endosc., vol. 69, no. 2, pp. 253-259, 2009.

[7] P. Swain, "The future of wireless capsule endoscopy," World J. Gastroenterol., vol. 14, no. 26, pp. 4142-4145, 2008.

[8] American Cancer Society. (2010, Feb.). [Online]. Available: http://www cancer.org/

[9] P. Dario, P. Ciarletta, A. Menciassi, and B. Kim, "Modelling and experimental validation of the locomotion of endoscopic robots in the colon,' Int. J. Robot. Res., vol. 23, no. 4-5, pp. 549-559, 2004.

[10] C. Stefanini, A. Menciassi, and P. Dario, "Modeling and experiments on a legged microrobot locomoting in a tubular, compliant and slippery environment," Int. J. Robot. Res., vol. 25, no. 5-6, pp. 551-560, 2006.

[11] X. Wang and M. Meng, "A magnetic stereo actuation mechanism for active capsule endoscope," in Proc. 29th Annu. Int. Conf. IEEE EMBS, 2007, pp. 2811-2814.

[12] F. Carpi, S. Galbiati, and A. Carpi, "Controlled navigation of endoscopic capsules: Concept and preliminary experimental investigations," IEEE Trans. Biomed. Eng., vol. 54, no. 11, pp. 2028-2036, Nov. 2007.

[13] F. Carpi and C. Pappone, "Magnetic manouvering of endoscopic capsules by means of a robotic navigation system," IEEE Trans. Biomed. Eng., vol. 56, no. 5, pp. 1482-1490, May 2009.

[14] F. Volke, A. Schneider, J. Gerber, M. Reimann-Zawadzki, E. Rebinovitz, C. Mosse, and P. Swain, "In vivo remote manipulation of modified capsule endoscopes using an external magnetic field," Gastrointest. Endosc., vol. A, pp. 121-122, 2008

[15] G. Ciuti, P. Valdastri, A. Menciassi, and P. Dario, "Robotic magnetic steering and locomotion of capsule endoscope for diagnostic and surgical endoluminal procedures," Robotica, vol. 28, pp. 199-207, 2010.

[16] M. Quirini, A. Menciassi, S. Scapellato, C. Stefanini, and P. Dario, "Design and fabrication of a motor legged capsule for the active exploration of the gastrointestinal tract," IEEE/ASME Trans. Mechatronics, vol. 13, no. 2, pp. 169-179, Apr. 2008.

[17] H. Park, S. Park, E. Yoon, B. Kim, J. Park, and S. Park, "Paddling based microrobot for capsule endoscopes," in Proc. IEEE Int. Conf. Robot. Autom., 10-14 Apr. 2007, pp. 3377-3382.

[18] P. Glass, M. Sitti, A. Pennathur, and R. Appasamy, "A swallowable tethered capsule endoscope for diagnosing barrett's esophagus," Gastrointest. Endosc., vol. 69, no. 5, p. AB106, Apr. 2009.

[19] W. Li, W. Guo, M. Li, and Y. Zhu, "A novel locomotion principle for endoscopic robot," in Proc. IEEE Int. Conf. Mechatronics Autom., Jun., 2006, pp. 1658-1662.

[20] B. Kim, S. Park, and J.-o. Park, "Microrobots for a capsule endoscope," in Proc. IEEE/ASME Int. Conf. Adv. Intell. Mechatronics, 14-17 Jul. 2009, pp. $729-734$. 
[21] M. Quirini, S. Scapellato, A. Menciassi, P. Dario, F. Rieber, C. N. Ho, S. Schostek, and M. O. Schurr, "Feasibility proof of a legged locomotion capsule for the GI tract," Gastrointest. Endosc., vol. 67, no. 7, pp. 11531158,2008

[22] P. Valdastri, R. J. Webster, C. Quaglia, M. Quirini, A. Menciassi, and P. Dario, "A new mechanism for meso-scale legged locomotion in compliant tubular environments," IEEE Trans. Robot., vol. 25, no. 5, pp. 10471057, Oct. 2009.

[23] D. Accoto, C. Stefanini, L. Phee, A. Arena, G. Pernorio, A. Menciassi, M. C. Carrozza, and P. Dario, "Measurements of tha frictional properties of the gastrointestinal tract," in Proc. World Tribol. Congr., 3-7 Sep. 2001, p. 728.

[24] M. Vatteroni, D. Covi, C. Cavallotti, P. Valdastri, A. Menciassi, P. Dario, and A. Sartori, "Smart optical CMOS sensor for endoluminal applications," Procedia Chem., vol. 1, no. 1, pp. 1271-1274, 2009.

[25] The VECTOR project website. (2010, Feb.). [Online]. Available: http:// www.vector-project.com/

[26] Namiki Precision Jewel Co., Ltd. (2010, Feb.). [Online]. Available: http://www.namiki.net

[27] Boston Gear Transmission Products. (2010, Feb.). [Online]. Available: http://www.bostongear.com/products/open/theory.html

[28] RoyMech Engineering Design. (2010, Feb.). [Online]. Available: http:// www.roymech.co.uk/Useful_Tables/Drive/Gears.html

[29] E. Buselli, P. Valdastri, M. Quirini, A. Menciassi, and P. Dario, "Superelastic leg design optimization for an endoscopic capsule with active locomotion," Smart Mater. Struct., vol. 18, no. 1, pp. 015001-1-015001-8, 2009.

[30] J. Agashe and D. Arnold, "A study of scaling and geometry effects on the forces between cuboidal and cylindrical magnets using analytical force solution," J. Phys. D, Appl. Phys., vol. 41, pp. 105001-1-105009, 2008.

[31] E. Susilo, P. Valdastri, A. Menciassi, and P. Dario, "A miniaturized wireless control platform for robotic capsular endoscopy using pseudokernel approach," Sens. Actuators A, vol. 156, pp. 49-58, 2009.

[32] P. Valdastri, A. Menciassi, and P. Dario, "Transmission power requirements for novel zigbee implants in the gastrointestinal tract," IEEE Trans. Biomed. Eng., vol. 55, no. 6, pp. 1705-1710, Jun. 2008.

[33] M. Jensen, J. Kanaley, J. Reed, and P. Sheedy, "Measurement of abdominal and visceral fat with computed tomography and dual-energy X-ray absorptiomentry," Amer. J. Clin. Nutr., vol. 61, pp. 274-278, 1995.

[34] American Society for Gastrointestinal Endoscopy. (2010, Feb.). [Online]. Available: http://www.askasge.org/

[35] R. Carta, G. Tortora, J. Thoné, B.Lenaers, P. Valdastri, A. Menciassi, P. Dario, and R. Puers, "Wireless powering for a self-propelled and steerable endoscopic capsule for stomach inspection," Biosens. Bioelectron. vol. 25 , no. 4 , pp. 845-851, 2009.

[36] M. Hocke, U. Schone, H. Richert, P. Gornert, J. Keller, P. Layer, and A. Stallmach, "Every slow-wave impulse is associated with motor activity of the human stomach," Amer. J. Physiol. Gastrointest. Liver Physiol., vol. 296, no. 4, pp. 709-716, 2008.

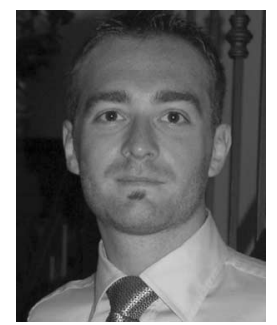

Massimiliano Simi received the Master's degree in biomedical engineering from the University of Pisa, Pisa, Italy, in April 2009. He is currently working toward the Ph.D. degree in biorobotics in the Center for Research in Microengineering (CRIM) Laboratory, Scuola Superiore Sant'Anna, Pisa.

He was engaged in the design, fabrication, and testing of an endocapsule with hybrid locomotion. Since June 2008, he has been with the CRIM Laboratory as a Research Assistant. His current research interests includes medical robotics and biomechatronics.

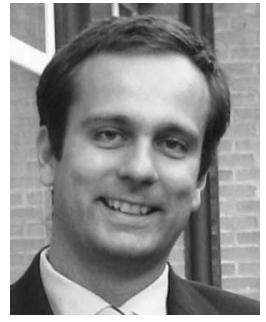

Pietro Valdastri (M'05) received the Master's (Hons.) degree in electronic engineering from the University of Pisa, Pisa, Italy, in 2002, and the Ph.D. degree in bioengineering from the Scuola Superiore Sant'Anna, Pisa, in 2006.

He is currently an Assistant Professor of biomedical robotics at the Scuola Superiore Sant'Anna, where he is engaged in research on implantable robotic systems and active capsule endoscopy. He is also involved in several European projects regarding the development of minimally invasive and wireless

biomedical devices.

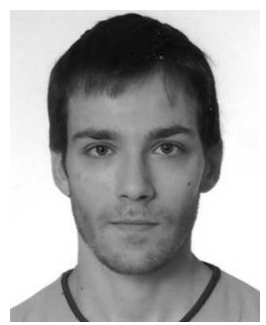

Claudio Quaglia (M'05) received the Master's degree in mechanical engineering from the University of Pisa, Pisa, Italy, in 2005. He is currently working toward the $\mathrm{Ph} . \mathrm{D}$. degree in microsystem engineering at the Scuola Superiore Sant'Anna, Pisa.

$\mathrm{He}$ was engaged in the employment of a triaxial accelerometer for tool condition monitoring in automatic machining. Since 2006, he has been with the Scuola Superiore Sant'Anna. His current research interests include medical robotics and micromechanical systems.

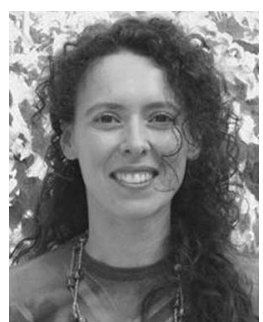

Arianna Menciassi (M'02) received the Master's degree in physics from the University of Pisa, Pisa, Italy, in 1995, and the Ph.D. degree in bioengineering from the Scuola Superiore Sant'Anna, Pisa, in 1999.

She is currently an Associate Professor of biomedical robotics at the Scuola Superiore Sant'Anna. She has authored or coauthored more than 130 international papers, about 70 in Institute for Scientific Information (ISI) journals, and five book chapters on medical devices and microtechnologies. Her research interests include biomedical micro- and nanorobotics for the development of innovative devices for surgery, therapy, and diagnostics.

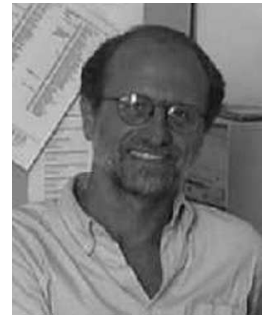

microendoscopy.

Mr. Dario is a recipient of the Joseph Engelberger Award as a Pioneer of Biomedical Robotics.

Paolo Dario (M'99-SM'01-F'03) received the Master's degree in mechanical engineering from the University of Pisa, Pisa, Italy, in 1977.

He is currently a Professor of biomedical robotics at the Scuola Superiore Sant'Anna, Pisa, where he supervises a team of about 150 young researchers. He has authored or coauthored more than 200 ISI journal papers, many international patents, and several book chapters on medical robotics. His research interests include biorobotics, mechatronic and robotic systems for rehabilitation, prosthetics, surgery, and 